\title{
Structure, Pose and Motion of Bilateral Symmetric Objects
}

\author{
T. N. Tan \\ Department of Computer Science \\ The University of Reading, Berkshire RG6 6AY, UK
}

Email: T.Tan@reading.ac.uk

\begin{abstract}
This paper concerns the determination of structure and pose of bilateral symmetric objects from a single perspective view. The estimation of 3-D inter-frame motion of such objects is also addressed. Particular attention is given to the interpretation of image-plane quadrilaterals as the perspective projection of bilateral symmetric trapezia. A simple algorithm is described for reconstructing a symmetric trapezium from a given image quadrilateral, and for determining 3-D motion from two matched image quadrilaterals. Experiments with both synthetic and real images are included to demonstrate the validity of the theoretic results.
\end{abstract}

\section{Introduction}

Symmetry can be found in many natural and man-made objects [13]. It provides strong constraints for human visual perception [1, 14]. An important form of symmetry is bilateral symmetry. The lines connecting the bilateral symmetric points are termed as symmetry lines. For perfect bilateral symmetry, the midpoints of all symmetry lines lie on the same line (called the symmetry axis for 2-D objects) or the same plane (called the symmetry plane for 3-D objects). Symmetry lines are parallel to each other, and are perpendicular to the symmetry axis or the symmetry plane.

Orthographic projection skews bilateral symmetry, leading to skewed symmetry. However, it preserves the parallelism and the midpoints of the symmetry lines. This is not the case under full perspective projection which in general causes the parallel symmetry lines to intersect at some vanishing point on the image plane. For convenience, we call the perspective projection of bilateral symmetry perspective symmetry.

Since Kanade's pioneering work on 3-D reconstruction using skewed symmetry [16], a plethora of approaches have been reported to skewed symmetry analysis [6-8, 10$12,15,17-18,25]$. These approaches typically focus on the detection of the axis of skewed symmetry. In contrast, work on perspective symmetry analysis has been more or less nonexistent until very recently. The best-known published work in this area includes Ulupinar and Nevatia [9], Mitsumoto et. al. [4], Glachet et. al. [3], and Rothwell et. al. [29]. These approaches typically make use of the vanishing point (VP) of the symmetry lines.

Unless the perspective distortion is severe, the detection of VPs is highly susceptible to image noise. A non-VP-based approach is therefore preferable in practice. In this paper, we present such an approach. We focus on the determination of structure and pose using a single perspective view. We also discuss the determination of 3-D inter- 
frame motion of bilateral symmetric objects - an area which has not been addressed by the existing work on perspective symmetry analysis. Since any two pairs of symmetric points of a bilateral symmetric object (either 2-D or 3-D) form a bilateral symmetric trapezium (simply called a trapezium hereafter), we concentrate in this paper on the determination of the structure and pose of a trapezium using a single view, and on the estimation of the 3-D inter-frame motion of the trapezium using two views. This not only allows us to focus on the basic issues of the problem but also helps gain more insight into the nature of the problem. The work on a trapezium presented in this paper can easily be extended to general bilateral symmetric objects. The extension will be reported in a future paper.

\section{Imaging geometry and notations}

The imaging geometry assumed in this paper is depicted in Fig.1. The camera coordinate

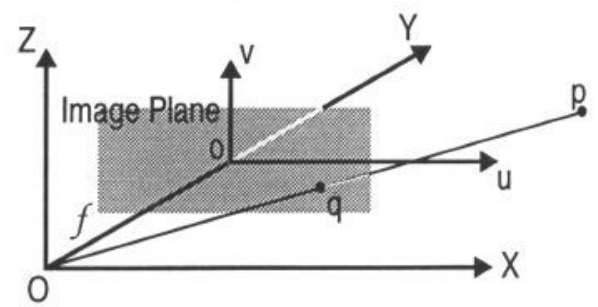

Figure 1: Illustration of imaging geometry.

system (CCS) is initially aligned with a world coordinate system (WCS). To allow the camera to view the 3-D world from an arbitrary viewpoint, the CCS is first rotated around the $\mathrm{X}$-axis by an angle $\phi$ (the tilt angle), then the Y-axis by an angle $\psi$ (the roll angle), and finally around the $\mathrm{Z}$-axis by an angle $\theta$ (the pan angle). The rotation is followed by a translation $T_{x}$ along the $\mathrm{X}$-axis, $T_{y}$ along the $\mathrm{Y}$-axis, and $T_{z}$ along the $\mathrm{Z}$-axis.

From now on, lowercase bold letters are used to denote (row) vectors. For example, we use $\boldsymbol{p}_{A}$ to represent the camera coordinates of a world point $A$, and $\boldsymbol{q}_{a}$ to represent the camera coordinates of the image $a$ of point $A$. If $a$ is located at $\left(u_{a}, v_{a}\right)$ on the image plane, $\boldsymbol{q}_{a}$ is given by $\boldsymbol{q}_{a}=\left(u_{a} f v_{a}\right)$, where $f$ is the known focal length. By definition, the focal point $O$, the image point $a$, and the world point $A$ are collinear on the line of sight. Given the image position of $a$, the direction of the line of sight is uniquely determined and is given by the following unit direction vector

$$
\hat{\boldsymbol{q}}_{a}=\boldsymbol{q}_{a} /\left|\boldsymbol{q}_{a}\right|
$$

Let the range of $A$ (the distance from the focal point) be $\lambda_{A}$. Then $p_{A}$ is given by

$$
p_{A}=\lambda_{A} \hat{\boldsymbol{q}}_{a}
$$

For two world points $A$ and $B$, the angle $\angle A O B$ is the same as the angle $\angle a O b$. Let the angle be denoted by $\alpha_{a b}\left(=\alpha_{b a}\right)$. Then we have

$$
\cos \alpha_{a b}=\hat{\boldsymbol{q}}_{a} \bullet \hat{\boldsymbol{q}}_{b} ; \quad \sin \alpha_{a b}=\sqrt{1-\cos ^{2} \alpha_{a b}}
$$

For a given trapezium, we name its four corners clockwise as $A, B, C$ and $D$, with $A$ and $B$, and $D$ and $C$ forming the symmetric pairs. The midpoints of the symmetry line 
segments $A B$ and $D C$ are labelled respectively as $E$ and $F$. We further denote the intersection of the two symmetric lines $A D$ and $B C$ by $G$ and that of the two diagonal lines $A C$ and $B D$ by $H$. The image of the trapezium is similarly labelled (see Fig.2). By

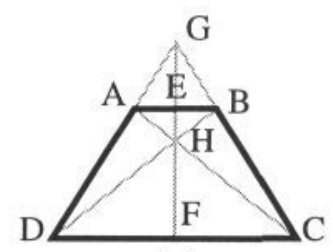

(a)

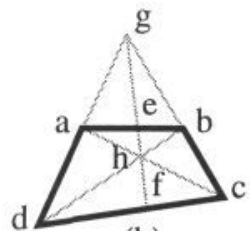

(b)

Figure 2: $\quad$ Labelling of a bilateral symmetric trapezium (a) and its image (b).

construction, points $G, E, H$ and $F$ are necessarily collinear and define the symmetry axis. Similarly, points $g, e, h$ and $f$ are collinear and define the projection of the axis.

In the next section, we start to discuss the determination of the range parameters $\lambda_{A}, \lambda_{B}, \lambda_{C}$ and $\lambda_{D}$ of the trapezium from a given image quadrilateral.

\section{Structure and pose determination}

For a given image quadrilateral $a b c d$, we assume known symmetric correspondences. In particular, $a b$ and $d c$ are assumed to be the projections of the symmetry lines. Since the midpoints of $a b$ and $d c$ are in general not the projections of the midpoints $E$ and $F$ of $A B$ and $D C$, the projections $e$ and $f$ of $E$ and $F$ are determined using the following simple proposition:

Prop 1 For a given image quadrilateral abcd, let the intersection of the two sides ad and $b c$ be $g$, and let that of the two diagonal lines ac and bd be $h$. Furthermore, let the intersections of line $g h$ with $a b$ and $d c$ be $e$ and $f$. Then there exists a symmetric trapezium $A B C D$ so that points $e$ and $f$ are the projections of the midpoints $E$ and $F$ of the two symmetry lines of the trapezium and abcd is the similar projection of $A B C D$.

The proof of the above proposition may be derived from the geometric construction and the illustration shown in Fig.2.

\subsection{Range determination}

Assume $e$ and $f$ have been determined using the above proposition. Let us now consider the triangle $\triangle A O B$ on the interpretation plane passing through the focal point $O$ and the image line segment $a b$. By definition, triangle $\triangle a O b$ is on the same plane. The geometric configuration is illustrated in Fig.3, where two parallel construction lines $A A^{\prime}$

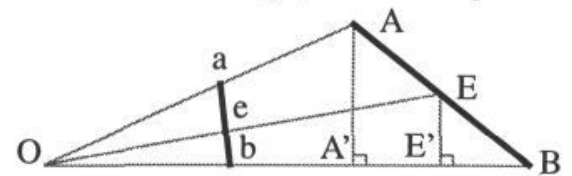

Figure 3: $\quad$ Triangles on an interpretation plane.

and $E E^{\prime}$ are also shown. The construction lines are perpendicular to the line of sight $O B$. By definition, we have 


$$
O A=\lambda_{A} ; \quad O E=\lambda_{E} ; \quad O B=\lambda_{B}
$$

The angles $\angle A O E\left(\alpha_{a e}\right), \angle B O E\left(\alpha_{b e}\right)$ and $\angle A O B\left(\alpha_{a b}\right)$ can easily be computed from the known image positions of $a, e$ and $b$ using (3).

From the right triangles $\triangle A A^{\prime} O$ and $\triangle E E^{\prime} O$, we get

$$
A A^{\prime}=O A \sin \angle A O B=\lambda_{A} \sin \alpha_{a b} ; E E^{\prime}=O E \sin \angle B O E=\lambda_{E} \sin \alpha_{b e}
$$

Since the two triangles $\triangle A A^{\prime} B$ and $\triangle E E^{\prime} B$ are similar, we have

$$
\frac{A A^{\prime}}{E E^{\prime}}=\frac{A B}{E B}=2
$$

The combination of (5)-(6) leads to an important equation:

$$
\lambda_{A}=\frac{2 \sin \alpha_{b e}}{\sin \alpha_{a b}} \lambda_{E} \equiv W_{A} \lambda_{E}
$$

By an identical process, we obtain a similar equation for $\lambda_{B}$

$$
\lambda_{B}=\frac{2 \sin \alpha_{a e}}{\sin \alpha_{a b}} \lambda_{E} \equiv W_{B} \lambda_{E}
$$

By considering the triangle $\triangle C O D$ and following the same procedure, we can express $\lambda_{C}$ and $\lambda_{D}$ in terms of $\lambda_{F}$ :

$$
\lambda_{C}=\frac{2 \sin \alpha_{d f}}{\sin \alpha_{c d}} \lambda_{F} \equiv W_{C} \lambda_{F} ; \quad \lambda_{D}=\frac{2 \sin \alpha_{c f}}{\sin \alpha_{c d}} \lambda_{F} \equiv W_{D} \lambda_{F}
$$

Since $E F \perp A B$, we have

$$
\left(\lambda_{E} \hat{\boldsymbol{q}}_{e}-\lambda_{F} \hat{\boldsymbol{q}}_{f}\right) \cdot\left(\lambda_{A} \hat{\boldsymbol{q}}_{a}-\lambda_{B} \hat{\boldsymbol{q}}_{b}\right)=0
$$

By substituting (7) and (8) into (10) and by re-arranging and combining terms, we get

$$
\lambda_{E}=\frac{W_{A} \cos \alpha_{a f}-W_{B} \cos \alpha_{b f}}{W_{A} \cos \alpha_{a e}-W_{B} \cos \alpha_{b e}} \lambda_{F} \equiv W \lambda_{F}
$$

Equations (7)-(11) simply state that the ranges of any five of the six points $A, B, C, D$, $E$ and $F$ can be expressed in terms of the range of the sixth point. This basically says that from a single view of a symmetric trapezium, the structure of the trapezium can be determined up to a global scale. For convenience, we take $F$ as the reference point and summarise (7)-(11) in the following

$$
\lambda_{A}=W_{A} W \lambda_{F} ; \quad \lambda_{B}=W_{B} W \lambda_{F} ; \quad \lambda_{C}=W_{C} \lambda_{F} ; \quad \lambda_{D}=W_{D} \lambda_{F} ; \quad \lambda_{E}=W \lambda_{F}
$$

The global scale in (12) may be fixed by knowing one of the following: a) the range of one of the points; b) the distance between any two of the points; or c) the area of the trapezium. Once the range parameters are determined, the camera coordinates of the six points are easily computed using (2). 


\subsection{Pose determination}

Once the camera coordinates of the trapezium are known, the estimation of the transformation (rotation and translation) from the WCS to the CCS is a simple matter. Assume the WCS is defined on the trapezium. The origin of the WCS is the midpoint of $D C$ (i.e., point $F$ ); the $+X_{w}$-axis is from $D$ to $C$, and the $+Y_{w}$-axis from $F$ to $E$; the $+Z_{w}$-axis is specified by the right-hand rule. Then the translation vector $t$ from the WCS to the CCS is simply the coordinates of point $F$ :

$$
\boldsymbol{t}=\left(\begin{array}{lll}
T_{x} & T_{y} & T_{z}
\end{array}\right)=\boldsymbol{p}_{F}=\lambda_{F} \hat{\boldsymbol{q}}_{f}
$$

To determine the rotation matrix, we first compute the unit direction vectors $\hat{\boldsymbol{x}}_{w}, \hat{\boldsymbol{y}}_{w}$ and $\hat{z}_{w}$ expressed in the CCS of the three axes of the WCS. They are given by

$$
\left\{\begin{array}{l}
\hat{\boldsymbol{x}}_{w}=\frac{\boldsymbol{p}_{C}-\boldsymbol{p}_{D}}{\left|\boldsymbol{p}_{C}-\boldsymbol{p}_{D}\right|}=\frac{W_{C} \lambda_{F} \hat{\boldsymbol{q}}_{c}-W_{D} \lambda_{F} \hat{\boldsymbol{q}}_{d}}{\left|W_{C} \lambda_{F} \hat{\boldsymbol{q}}_{c}-W_{D} \lambda_{F} \hat{\boldsymbol{q}}_{d}\right|}=\frac{W_{C} \hat{\boldsymbol{q}}_{C}-W_{D} \hat{\boldsymbol{q}}_{d}}{\left|W_{C} \hat{\boldsymbol{q}}_{C}-W_{D} \hat{\boldsymbol{q}}_{d}\right|} \\
\hat{\boldsymbol{y}}_{w}=\frac{\boldsymbol{p}_{E}-\boldsymbol{p}_{F}}{\left|\boldsymbol{p}_{E}-\boldsymbol{p}_{F}\right|}=\frac{W \lambda_{F} \hat{\boldsymbol{q}}_{e}-\lambda_{F} \hat{\boldsymbol{q}}_{f}}{\left|W \lambda_{F} \hat{\boldsymbol{q}}_{e}-\lambda_{F} \hat{\boldsymbol{q}}_{f}\right|}=\frac{W \hat{\boldsymbol{q}}_{e}-\hat{\boldsymbol{q}}_{f}}{\left|W \hat{\boldsymbol{q}}_{e}-\hat{\boldsymbol{q}}_{f}\right|} \\
\hat{z}_{w}=\hat{\boldsymbol{x}}_{w} \times \hat{\boldsymbol{y}}_{w}
\end{array}\right.
$$

Then the rotation matrix $\boldsymbol{R}$ from the WCS to the CCS is simply the following

$$
\boldsymbol{R}=\left[\begin{array}{lll}
\hat{\boldsymbol{x}}_{w}^{T} & \hat{\boldsymbol{y}}_{w}^{T} & \hat{z}_{w}^{T}
\end{array}\right]
$$

It is worth noting that the rotation matrix given in (14)-(15) is independent of the global scale. This implies that the camera viewing angles can be determined from a single perspective view of a symmetric trapezium of unknown size. This observation is more general than Haralick's [2], where he noted that a single perspective view of a rectangle (which is a special symmetric trapezium) of unknown size is sufficient to determine the camera viewing angles.

\subsection{Structure and pose with unknown symmetric correspondences}

It has so far been assumed that the symmetric correspondences are known. Without such a priori knowledge, there are four sets of solutions to consider corresponding to the four different labellings of the corners. An example is given in Fig.4 to elucidate the case. Fig.4(a) shows the original symmetric trapezium with given size. Fig.4(b)-(e) are the four

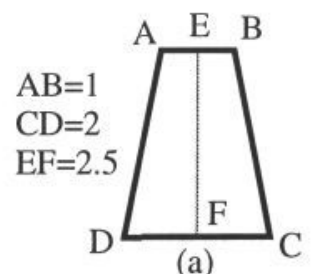

(a)
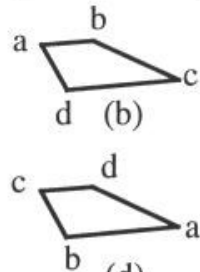

(d)

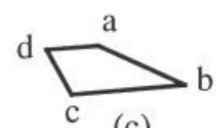

(c)

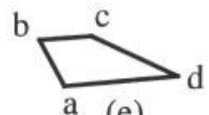

(e)

Figure 4: Illustration of a bilateral symmetric trapezium ((a)) and four labellings $((b)-(e))$ of its perspective image. 
different labellings of its perspective image, with Fig.4(b) being the correct labelling. The corresponding structures and poses recovered are summarised in Table 1, where the

\begin{tabular}{|c|c|c|c|c|c|c|c|c|c|c|}
\hline $\begin{array}{c}\text { Label- } \\
\text { ling }\end{array}$ & AB & CD & EF & Tilt & Roll & Pan & Tx & Ty & Tz & $\begin{array}{c}\text { Com- } \\
\text { pactness }\end{array}$ \\
\hline \hline Fig.4(b) & 1.00 & 2.00 & 2.50 & 14.29 & -3.77 & 19.97 & 1.48 & 23.57 & -1.39 & 0.057 \\
\hline Fig.4(c) & 7.46 & 7.60 & 0.50 & 4.85 & -0.32 & -89.50 & 0.11 & 10.08 & -0.41 & 0.015 \\
\hline Fig.4(d) & 2.00 & 1.00 & 2.50 & -14.29 & 3.77 & -160.03 & 0.62 & 25.83 & -0.77 & 0.057 \\
\hline Fig.4(e) & 7.60 & 7.46 & 0.50 & -4.85 & 0.32 & 90.50 & 0.61 & 10.08 & -0.37 & 0.015 \\
\hline
\end{tabular}

Table 1: Recovered structure and pose for the trapezium shown in Fig.4.

global scale in each case is fixed by using the same known area of the trapezium. The structure and pose recovered under the correct correspondence (Fig.4(b)) are exactly the same as the ground-truth. This demonstrates the validity of the algorithm.

The correct solution may be selected from the four candidates using the extremum principle as proposed by Brady and Yuille [5]. For example, one can choose the candidate solution which results in the maximal compactness of the recovered trapezium, where compactness is defined as the area divided by the squared perimeter of the trapezium. The compactness measures so computed for the four solutions are listed in the last column of Table 1. It can be seen that the correct labelling Fig.4(b) has a much higher compactness measure than the two wrong labellings shown in Fig.4(c) and (e). It should also be noted that the labelling in Fig.4(d) leads to a trapezium having the same compactness as that derived from Fig.4(b). Therefore the labellings shown in Fig.4(b) and (d) cannot be distinguished based on the compactness measure. This ambiguity is similar to the ambiguity in the perception of the Necker cube [26]. Experiments with a variety of symmetric trapezia and viewpoints have confirmed that the maximal compactness principle can be used to select the correct interpretation up to the Necker reversal.

\subsection{Experiments with real images}

The algorithm described in this section was also applied to real CCD camera images. A typical example is shown in Fig.5. The image contains the perspective view of six

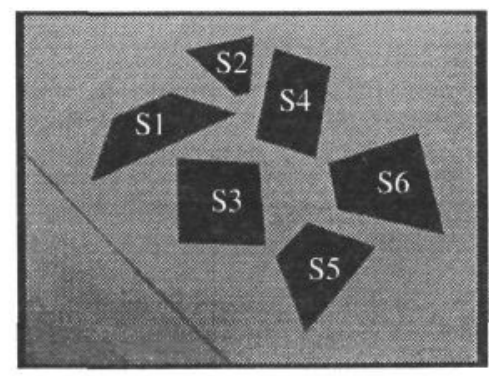

Figure 5: An image showing the perspective view of six trapezia. The image is of size $768 \times 576$ pixels. 
symmetric trapezia, with one (S3) being a square and one (S4) a rectangle. The image was first processed by the Plessey corner detector [27] to find the six quadrilaterals (the grouping of corners into quadrilaterals was done manually). The quadrilaterals were then interpreted by the algorithm by assuming known symmetric correspondences.

The true and the recovered sizes of the trapezia are listed in Table 2, where w1

\begin{tabular}{|c|c|c|c|c|c|c|}
\hline & \multicolumn{3}{|c|}{ True Structure (mm) } & \multicolumn{2}{c|}{ Recovered Structure (mm) } \\
\hline \hline Shape & w1 & w2 & w3 & w1 & w2 & w3 \\
\hline S1 & 40.00 & 100.00 & 30.00 & 40.00 & 99.81 & 28.98 \\
\hline S2 & 10.00 & 50.00 & 40.00 & 10.00 & 48.75 & 38.34 \\
\hline S3 & 50.00 & 50.00 & 50.00 & 50.00 & 50.07 & 49.98 \\
\hline S4 & 70.00 & 70.00 & 40.00 & 70.00 & 69.68 & 39.60 \\
\hline S5 & 30.00 & 70.00 & 40.00 & 30.00 & 68.60 & 40.13 \\
\hline S6 & 30.00 & 70.00 & 70.00 & 30.00 & 70.74 & 68.80 \\
\hline
\end{tabular}

Table 2 : True and recovered structures of six trapezia shown in Fig.5.

and w2 are the length of the two symmetry line segments, and w3 the height of a trapezium. The global scale was fixed in each trapezium by assuming known w1. It can be seen that in all cases, the recovered sizes are very close to the ground-truth, further demonstrating the validity of the algorithm.

\section{Inter-frame motion estimation}

We now turn our attention to the determination of 3-D inter-frame motion of a symmetric trapezium from two image quadrilaterals obtained at two time instants. We assume known intra-frame symmetric correspondences and inter-frame point correspondences. The problem is the well-known structure-from-motion (SFM) problem and may be solved using one of the many existing methods $[19,28]$.

The two matched quadrilaterals provide four pairs of point correspondences in two frames. Without additional constraints, four points in two frames are inadequate for SFM. For example, for general point configurations, a minimum of 8 points in two frames is required to allow a linear closed-form solution [28]. When the coplanarity of the four corner points of the image quadrilaterals is explicitly considered, the problem becomes solvable but the solutions are not unique [21]. The solvability of the problem may be explained in a different way. Two views of a symmetric trapezium are equivalent to four views of half of the trapezium. Hence the four point correspondences in two frames can be regarded as two point correspondences in four frames. With two points in four frames, the structure and motion parameters may be recovered by solving a set of non-linear equations [20]. The solution is however rather indirect and cumbersome.

By using the algorithm presented in the previous section, the 3-D motion of a symmetric trapezium can be determined in a very simple and efficient way. For each 
view, the image quadrilateral is interpreted by the algorithm to determine the camera coordinates of the four corners of the trapezium. The global scale in each frame may be resolved by assuming the same area of the trapezium so as to ensure consistent inter-frame scale. At the end of this process, we obtain four point correspondences with known 3-D coordinates. The 3-D inter-frame motion can then easily be determined by using one of the standard absolute orientation algorithms [24]. Several of these algorithms, e.g.. Arun, Huang and Blostein [22], and Horn [23], provide efficient closed-form solutions.

A typical example with real CCD camera images is given in Fig.6 to illustrate

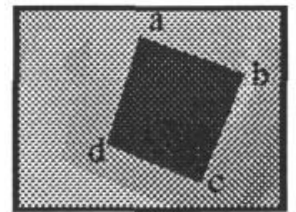

(a)

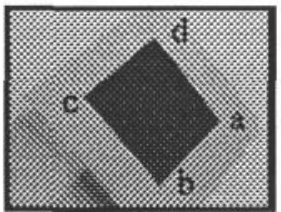

(b)

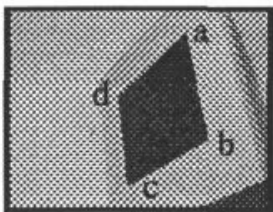

(c)

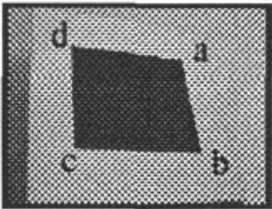

(d)

Figure 6: Four perspective views of a rectangle. Images are of size $768 \times 576$ pixels.

the ideas outlined above. The images in Fig.6 show four views of a trapezium (which is in fact a rectangle). The images were first processed by the Plessey corner detector to locate the image quadrilaterals. The four quadrilaterals were interpreted independently by the algorithm of Section 4 to determine the camera coordinates of the four corners. Horn's algorithm [23] was then applied to the known coordinates to estimate the motion between the reference frame shown in Fig.6(a) and each of the other three frames.

Since true motion parameters were unknown, the following procedure was adopted to assess the accuracy of the recovered motion. The camera coordinates of the four corners in the three frames shown in Fig.6(b)-(d) were predicted based on the recovered motion and the recovered coordinates of the four corners in the reference frame. Image positions of the four corners in the three frames were reconstructed from the predicted coordinates. The reconstructed positions were then compared with the original image positions. The results are shown in Table 3 . In all cases, the average absolute error

\begin{tabular}{|c|c|c|c|c|c|c|}
\hline \multirow{2}{*}{ Frame } & & a & b & c & d & $\begin{array}{c}\text { Av. } \\
\text { Err. }\end{array}$ \\
\hline \hline \multirow{2}{*}{ Fig.6(b) } & Original & 617,320 & 442,509 & 225,249 & 425,83 & - \\
\cline { 2 - 7 } & Recovered & $616.97,320.89$ & $442.91,508.96$ & $224.87,248.24$ & $424.38,82.73$ & 0.81 \\
\hline \multirow{2}{*}{ Fig.6(c) } & Original & 517,68 & 585,375 & 349,511 & 322,248 & - \\
\cline { 2 - 7 } & Recovered & $516.54,68.03$ & $584.62,375.73$ & $349.35,511.41$ & $322.2,247.32$ & 0.63 \\
\hline \multirow{2}{*}{ Fig.6(d) } & Original & 514,154 & 571,422 & 203,411 & 194,115 & - \\
\cline { 2 - 7 } & Recovered & $514.34,154.39$ & $571.2,421.53$ & $202.62,410.89$ & $193.68,115.03$ & 0.44 \\
\hline
\end{tabular}

Table 3 : Image positions (pixels) reconstructed based on recovered motion.

(distance) between the original and the reconstructed image positions is less than 1 pixel, indicating good accuracy of the recovered motion parameters. 


\section{Conclusions}

The determination of structure and pose of bilateral symmetric objects from a single view has been addressed in this paper. The estimation of 3-D inter-frame motion has also been discussed. A simple algorithm has been described for reconstructing a bilateral symmetric trapezium from a given image quadrilateral in closed-form. The algorithm facilitates simple and efficient determination of 3-D motion of bilateral symmetric objects.

The paper has established and/or further verified the following results:

- The camera viewing angles are computable from a single perspective view of a bilateral symmetric trapezium of unknown size.

- When symmetric correspondences are known, the interpretation of an image quadrilateral as the projection of a symmetric trapezium is unique.

- When symmetric correspondences are unknown, there are four possible interpretations of an image quadrilateral as the projection of a symmetric trapezium. The maximal compactness principle can be used to determine the correct interpretation up to the Necker reversal.

- Bilateral symmetry can be exploited to allow simple and efficient motion estimation.

\section{References}

[1] J. Wagemans, et. al., Symmetry Detection in Human Vision, Proc. of SPIE, vol.1099, 1989, pp.44-55.

[2] R. M. Haralick, Determining Camera Parameters from the Perspective Projection of a Rectangle, Pattern Recognition, vol.22, 1989, pp.225-230.

[3] R. Glachet, et. al., Locating and Modelling a Flat Symmetric Object from a Single Perspective Image, CVGIP: Image Understanding, vol.57, 1993, pp.219-226.

[4] H. Mitsumoto, et. al., 3-D Reconstruction Using Mirror Images Based on a Plane Symmetry Recovering Method, IEEE Trans. PAMI, vol.14, 1992, pp.941-946.

[5] J. M. Brady and A. Yuille, An Extremum Principle for Shape from Contour, IEEE Trans. PAMI, vol.6, 1984, pp.288-301.

[6] S. A. Friedberg, Finding axes of skewed symmetry, CVGIP, vol.34, 1986, pp.138-155.

[7] A. D. Gross and T. E. Boult, Analysing Skewed Symmetries, Int. J. Computer Vision, vol.13, 1994, pp.91-111.

[8] J. Ponce, On Characterizing Ribbons and Finding Skewed Symmetries, CVGIP, vol.52, 1990, pp.328-340.

[9] F. Ulupinar and R. Nevatia, Constraints for Interpretation of Line Drawings Under Perspective Projection, Image Understanding, vol.53, 1991, pp.88-96.

[10] S. Y. Yuen, Shape from Contour Using Symmetries, Proc. of Ist European Conf. Comput. Vision, Antibes, France, April 1990, pp.437-453.

[11] P. L. Palmer, M. Petrou and J. Kittler, An Optimisation Approach to Improve the Accuracy of the Hough Transform - Plane Orientations from Skewed Symmetry, Proc. of CVPR93, pp.682-683. 
[12] S. Posch, Detecting Skewed Symmetries, Proc. of 11th Int. Conf. Pattern Recognition, vol.III, 1992, pp.602-606.

[13] H. Weyl, Symmetry, Princeton University Press, 1952.

[14] M. Enquist and A. Arak, Symmetry, Beauty and Evolution, Nature, vol.372, 1994, pp.169-172.

[15] R. Fawcett, et al., Extracting Structure from an Affine View of a 3D Point Set with One or Two Bilateral Symmetries, Proc. of BMVC93, vol.2, 1993, pp.349-350.

[16] T. Kanade, Recovery of the Three-Dimensional Shape of an Object from a Single View, Artificial Intelligence, vol.17, 1981, pp.409-460.

[17] D.P.Mukherjee et al., Shape from Symmetry: Detecting and Exploiting Symmetry in Affine Images, Phil. Trans. R. Soc. Lond. A, vol.351, 1995, pp.77-106.

[18] T. J. Cham and R. Cipolla, Skewed Symmetry Detection Through Local Skewed Symmetries, Proc. of BMVC94, vol.2, 1994, pp.549-558.

[19] T.N.Tan, G.D.Sullivan and K.D.Baker, 3D Structure and Motion Estimation from 2D Image Sequences, Image and Vision Computing, vol.11, 1993, pp.203-210.

[20] H. Shariat and K. E. Price, Motion Estimation with More than Two Frames, IEEE Trans. PAMI, vol.12, 1990, pp.417-433.

[21] J. Weng, N. Ahuja and T. S. Huang, Motion and Structure from Point Correspondences with Error Estimation: Planar Surfaces, IEEE Trans. Signal Processing, vol.39, 1991, pp.2691-2716.

[22] K. Arun, T. S. Huang and S. Blostein, Least Squares Fitting of Two 3-D Point Sets, IEEE Trans. PAMI, vol.9, 1987, pp.698-700.

[23] B. Horn, Closed-form Solution of Absolute Orientation Using Unit Quaternion, J. Opt. Soc. Am. A, vol.4, 1987, pp.629-642.

[24] B. Sabata and J. K. Aggarwal, Estimation of Motion from A Pair of Range Images: A Review, CVGIP: Image Understanding, vol.54, 1991, pp.309-324.

[25] H. Zabrodsky and D. Weinshall, Utilizing Symmetry in the Reconstruction of Three-Dimensional Shape from Noisy Images, Proc. of 3rd European Conf. Comput. Vision, Sweden, May 1994, pp.403-410.

[26] M. A. Fischler and O. Firschein, Intelligence: The Eye, the Brain, and the Computer, Addison-Wesley, 1987.

[27] J. A. Noble, Finding Corners, Proc. of 3rd Alvey Vision Conf., Cambridge, England, September 1987, pp.267-274.

[28] T. S. Huang and A. N. Netravali, Motion and Structure from Feature Correspondences: A Review, Proc. of IEEE, vol.82, 1994, pp.252-268.

[29] C. A. Rothwell et. al., Extracting Projective Structure from Single Perspective Views of 3-D Point Sets, Proc. of 4th ICCV, Berlin, Germany, 1993, pp.573-582. 